hep-th/0306145

\title{
Free tensor multiplets and strings in spontaneously broken six-dimensional $(2,0)$ theory
}

\author{
Pär Arvidsson ${ }^{1}$, Erik Flink ${ }^{2}$ and Måns Henningson ${ }^{3}$ \\ Department of Theoretical Physics \\ Chalmers University of Technology and Göteborg University \\ SE-412 96 Göteborg, Sweden
}

\begin{abstract}
We first review the representations of the six-dimensional $(2,0)$ superalgebra on a free tensor multiplet and on a free string. We then construct a supersymmetric Lagrangian describing a free tensor multiplet. (It also includes a decoupled anti self-dual part of the three-form field strength.) This field theory is then rewritten in variables appropriate for analyzing a situation where the $R$-symmetry is spontaneously broken by the vacuum expectation values of the scalar moduli fields. Finally, we construct a supersymmetric and $\kappa$-symmetric action for a free string.
\end{abstract}

\footnotetext{
${ }^{1}$ par@fy.chalmers.se

2erik.flink@fy.chalmers.se

${ }^{3}$ mans@fy.chalmers.se
} 


\section{Introduction}

In a previous publication [1], we outlined a programme to systematically study the six-dimensional $(2,0)$ theories, with the ultimate goal of eventually finding an intrinsic definition of them that does not rely on their embedding into string or $M$-theory. The $(2,0)$ theories obey an ADE-classification, but have no other discrete or continuous parameters 2. However, such a theory has a moduli space of inequivalent vacuum states, parametrized by the expectation values of a set of scalar fields. At the origin of the moduli space, the theory is given by some strongly coupled superconformal quantum theory with a global $R$-symmetry group $G \simeq S O(5)$. In a certain sense, the most natural way to study a $(2,0)$ theory would be to work directly around this point in the moduli space. However, although some intriguing properties of these superconformal theories are known, it seems fair to say that they are still rather mysterious and, with present techniques, not easily studied.

Our approach is instead based on the idea of working at a generic point in the moduli space, where the $R$-symmetry is spontaneously broken to a subgroup $H$ isomorphic to $S O(4)$. Here, a $(2,0)$ theory describes massless particles interacting with tensile strings. The particles can be obtained by quantizing a set of six-dimensional fields known as a $(2,0)$ tensor multiplet. The tensor multiplet comprises the scalar fields, whose expectation values parametrize the moduli space, and their superpartners. The distance to the origin of the moduli space determines the tension of the strings, whereas the remaining scalar fields can be interpreted as the Goldstone bosons associated with the spontaneous breaking of the $R$-symmetry group from $G \simeq S O(5)$ to $H \simeq S O(4)$. (For simplicity, we limit ourselves in this discussion to the $A_{1}$ version of $(2,0)$ theory, which only contains a single tensor multiplet and a single type of string.) On the world-sheet of a string, we have a set of two-dimensional fields consisting of the Goldstone bosons and fermions associated with the further spontaneous breaking of the $(2,0)$ superalgebra by the string.

The dynamics of the theory can be investigated by defining a dimensionless parameter as the typical energy scale of some process, e.g. the energy of an incoming tensor multiplet quantum, divided by the square root of the string tension. In the limit that the string tension goes to infinity, so that this parameter goes to zero, the six-dimensional tensor multiplet fields propagating in space-time and the twodimensional fields propagating on the world-sheets of the strings decouple from each other. The ensuing free theory is exactly solvable and can serve as a starting point for a perturbative analysis of the interacting theory that is obtained at a finite string tension.

In this paper, we continue our work along these lines. In the next section, we review the $(2,0)$ superalgebra and its representations on particles and strings. In section three, we give a Lagrangian for a free tensor multiplet, which to our knowledge has not yet appeared in the literature. In section four, we rewrite this field theory in a way appropriate for working at a generic point in the moduli space with spontaneously broken $R$-symmetry. Finally, in section five, we construct the theory of a free 
string. In a forthcoming publication, we intend to pursue the challenging problem of constructing the supersymmetric theory of tensor multiplet particles interacting with tensile, self-dual strings. We hope that the results of the present paper will provide a firm basis for this project.

\section{The (2,0) superalgebra and its representations}

The $(2,0)$ superalgebra [3] contains the six-dimensional Lorentz algebra $s o(5,1)$ as a subalgebra. The basic representations of the latter algebra, from which all other representations can be constructed, are the Weyl spinor $\mathbf{4}$ and the anti-Weyl spinor $4^{\prime}$. We denote quantities transforming in these representations with a subscript or superscript index $\alpha=1, \ldots, 4$ respectively. The tensor products of two of these representations are

$$
\begin{aligned}
\mathbf{4} \otimes \mathbf{4} & \simeq \mathbf{6} \oplus \mathbf{1 0 _ { + }} \\
\mathbf{4}^{\prime} \otimes \mathbf{4}^{\prime} & \simeq \mathbf{6} \oplus \mathbf{1 0}{ }_{-} \\
\mathbf{4} \otimes \mathbf{4}^{\prime} & \simeq \mathbf{1} \oplus \mathbf{1 5} .
\end{aligned}
$$

Here, $\mathbf{1}$ is of course the singlet, associated with the invariant tensor $\delta_{\alpha}{ }^{\beta}$. The $\mathbf{6}$ is the vector, $\mathbf{1 0}_{+}$and $\mathbf{1 0}$ - are the self-dual and anti self-dual three-forms respectively, and $\mathbf{1 5}$ is the two-form representation of $s o(5,1)$. Finally, we note that the completely antisymmetric tensor product of four Weyl spinors or of four anti-Weyl spinors is a singlet, and we denote the corresponding invariant tensors as $\epsilon_{\alpha \beta \gamma \delta}$ and $\epsilon^{\alpha \beta \gamma \delta}$ respectively.

The $(2,0)$ superalgebra also has an $R$-symmetry subalgebra isomorphic to $s o(5)$. For our purposes, it is convenient to think of $s o(5)$ as a subalgebra of $s o(6)$, and use the fact that $s o(6) \simeq s u(4)$. In terms of Lie groups, $S O(6) \simeq S U(4) / \mathbb{Z}_{2}$. An element $g \in S U(4)$ is a four-by-four complex matrix such that $\operatorname{det} g=1$ and $g g^{\dagger}=\mathbf{1}_{4}$. In our case, the most important representations of $S U(4)$, apart from the singlet $\mathbf{1}$, are the 4, the $\mathbf{6}$, and the 10. They correspond to a four-by-one column vector $\Psi$, an antisymmetric four-by-four matrix $A$, and a symmetric four-by-four matrix $S$ respectively. An element $g \in S U(4)$ acts on these as

$$
\begin{aligned}
& \Psi \rightarrow \Psi^{\prime}=g \Psi \\
& A \rightarrow A^{\prime}=g A g^{T} \\
& S \rightarrow S^{\prime}=g S g^{T} .
\end{aligned}
$$

Now choose a fixed element $\Omega$ in the 6 representation of $S U(4)$, i.e. an antisymmetric four-by-four matrix, and normalize it so that $-\frac{1}{4} \operatorname{Tr}\left(\Omega \Omega^{*}\right)=1$. Explicitly, we take

$$
\Omega=\left(\begin{array}{cc}
\sigma_{2} & \mathbf{0} \\
\mathbf{0} & \sigma_{2}
\end{array}\right)=\left(\begin{array}{cccc}
0 & -i & 0 & 0 \\
i & 0 & 0 & 0 \\
0 & 0 & 0 & -i \\
0 & 0 & i & 0
\end{array}\right)
$$


We can then define a subgroup $G \simeq S O(5)$ in $S U(4)$ by the requirement that $g \in G$ if and only if $g \Omega g^{T}=\Omega$. Hence $\Omega$ itself is in the singlet representation of $G$. We also get a five-dimensional representation of $G$ on the set of anti-symmetric four-by-four matrices $\Phi$ obeying $\operatorname{Tr}\left(\Omega \Phi^{*}\right)=0$, so the 6 representation of $S U(4)$ decomposes into the irreps $\mathbf{1}$ and $\mathbf{5}$ of the subgroup $G$. The $\mathbf{4}$ representation of $S U(4)$ is still irreducible under $G$, and the tensor product of two such representations is given by

$$
\mathbf{4} \otimes \mathbf{4} \simeq \mathbf{1} \oplus \mathbf{5} \oplus \mathbf{1 0}
$$

We are now ready to discuss the complete $(2,0)$ superalgebra. Its fermionic generators $Q_{\alpha}$ transform in the Weyl representation 4 under the Lorentz group and in the 4 representation under the $G \simeq S O(5) R$-symmetry group. They also obey the symplectic Majorana reality condition [4]

$$
Q_{\alpha}^{*}=C_{\alpha}^{\beta} \Omega Q_{\beta} .
$$

In this relation, the charge conjugation operator $C_{\alpha}{ }^{\beta}$ is an $S O(5)$ scalar obeying

$$
C^{*}{ }_{\alpha}^{\beta} C_{\beta}^{\gamma}=-\delta_{\alpha}^{\gamma},
$$

while $\Omega$ is the $S O(5)$ invariant matrix introduced above. Later, we will also use quantities, such as the parameters $\eta^{\alpha}$ of infinitesimal supersymmetry transformations, that transform in the anti-Weyl representation $4^{\prime}$ under the Lorentz group and in the 4 representation under $R$-symmetry. Here we introduce the similar reality condition

$$
\eta^{* \alpha}=-C_{\beta}^{*}{ }^{\alpha} \Omega \eta^{\beta} \text {. }
$$

According to the above, the anti-commutator of two fermionic generators must be of the form [ $[$ ]

$$
\left\{Q_{\alpha}, Q_{\beta}^{T}\right\}=P_{\alpha \beta} \Omega+Z_{\alpha \beta}+W_{\alpha \beta} .
$$

Here, $P_{\alpha \beta}=P_{[\alpha \beta]}$ are scalars under $G \simeq S O(5)$, and are interpreted as the sixdimensional momentum operators. The generators $Z_{\alpha \beta}=Z_{[\alpha \beta]}$ transform in the $\mathbf{5}$ representation of $G$, i.e. $Z_{\alpha \beta}=-Z_{\alpha \beta}^{T}$ and $\operatorname{Tr}\left(\Omega Z_{\alpha \beta}^{*}\right)=0$. They are 'central charges' that appear in the presence of strings. Finally, the generators $W_{\alpha \beta}=W_{(\alpha \beta)}$ form another set of central charges (associated with self-dual three-brane solitons) that transform in the 10 representation of $G$, i.e. $W_{\alpha \beta}=W_{\alpha \beta}^{T}$. The bosonic subalgebra of the $(2,0)$ superalgebra is spanned by $P_{\alpha \beta}, Z_{\alpha \beta}$ and $W_{\alpha \beta}$ together with the Lorentz generators and the $R$-symmetry generators.

When constructing an irreducible representation of the $(2,0)$ superalgebra, we may impose any $S O(5,1) \times S O(5)$ covariant conditions on the eigenvalues of the simultaneously diagonalizable generators $P_{\alpha \beta}, Z_{\alpha \beta}$ and $W_{\alpha \beta}$. For example, we may consider the case when the momentum $P_{\alpha \beta}$ is restricted to be light-like and the central charges $Z_{\alpha \beta}$ and $W_{\alpha \beta}$ vanish. Such a configuration spontaneously breaks the Lorentz group to the little subgroup $S O(4) \simeq S U(2) \times S U(2)$, but leaves the $R$-symmetry 
group $S O(5)$ unbroken. The supersymmetry generators transform under $S O(4) \times$ $S O(5) \simeq S U(2) \times S U(2) \times S O(5)$ as

$$
(1,2 ; 4) \oplus(2,1 ; 4) .
$$

It can be seen from the supersymmetry algebra that the supercharges of the first term are unbroken and annihilate a quantum state of this configuration. The supercharges of the second term form a Clifford algebra, which can be represented on a set of states transforming as

$$
(3,1 ; 1) \oplus(2,1 ; 4) \oplus(1,1 ; 5) .
$$

These $8+8$ states are known as a $(2,0)$ tensor particle multiplet, because of the states $(\mathbf{3}, \mathbf{1} ; \mathbf{1})$, that transform in the rank two antisymmetric self-dual tensor representation of the unbroken $S O(4)$ subgroup of the Lorentz group.

Another set of conditions can be described as follows [6] The central charge $Z_{\alpha \beta}$ is a tensor product of a space-like Lorentz vector $V_{\alpha \beta}$ invariant under $R$-symmetry, and a Lorentz scalar $\Phi$ in the $\mathbf{5}$ representation of the $R$-symmetry group. The central charge $W_{\alpha \beta}$ vanishes. The scalar product of $P_{\alpha \beta}$ and $Z_{\alpha \beta}$ is also zero. These conditions describe a configuration with a straight infinite string pointing in the direction of $V_{\alpha \beta}$ in a background with the moduli given by $\Phi$. Unitarity of the representation imposes that $P_{\alpha \beta}$ be time-like with a certain lower bound on the energy (per unit length of the string). We take $P_{\alpha \beta}$ to saturate this bound. A non-zero $\Phi$ breaks the $R$-symmetry group spontaneously to $S O(4) \simeq S U(2) \times S U(2)$, and the vectors $P_{\alpha \beta}$ and $V_{\alpha \beta}$ break the Lorentz group to $S O(4) \simeq S U(2) \times S U(2)$. The supersymmetry generators transform as

$$
(2,1 ; 2,1) \oplus(1,2 ; 1,2) \oplus(2,1 ; 1,2) \oplus(1,2 ; 2,1),
$$

under $S O(4) \times S O(4) \simeq S U(2) \times S U(2) \times S U(2) \times S U(2)$, where the first $S O(4)$ factor comes from the Lorentz group and the second from the $R$-symmetry group. It can be seen from the supersymmetry algebra that the supercharges of the last two terms are unbroken and annihilate such a state. The supercharges of the first two terms form a Clifford algebra, which can be represented on a set of states transforming as

$$
(\mathbf{2}, \mathbf{2} ; \mathbf{1}, \mathbf{1}) \oplus(\mathbf{2}, \mathbf{1} ; \mathbf{1}, \mathbf{2}) \oplus(\mathbf{1}, \mathbf{2} ; \mathbf{2}, \mathbf{1}) \oplus(\mathbf{1}, \mathbf{1} ; \mathbf{2}, \mathbf{2}) .
$$

We call this set of $8+8$ states a $(2,0)$ vector string multiplet, because of the states $(\mathbf{2}, \mathbf{2} ; \mathbf{1}, \mathbf{1})$ that transform as a vector under the unbroken $S O(4)$ subgroup of the Lorentz group.

\section{The tensor multiplet field theory}

\subsection{The fields}

The Fock space of the tensor particle multiplet described in the previous section may be obtained by quantizing a free field theory. We will start by defining the field content of this theory. 
First, we have a set of fermionic fields which are both Lorentz and $R$-symmetry spinors, i.e. transform in the 4 representation of $S O(5,1)$ and in the 4 representation of $S O(5)$. These are written as (Grassmann odd) column vectors $\Psi_{\alpha}$ with four complex components and obey the symplectic Majorana condition as defined in Eq. (7).

Next, we have a set of bosonic fields in the $\mathbf{5}$ (vector) representation of $S O(5)$, transforming as Lorentz scalars. These fields are represented by the four-by-four antisymmetric matrices $\Phi$ described above (obeying $\operatorname{Tr}\left(\Omega \Phi^{*}\right)=0$ ) and are subject to the reality condition

$$
\Phi^{*}=-\Omega \Phi \Omega .
$$

The vacuum expectation values of these fields constitute the moduli of the theory.

Finally, we have a two-form gauge field in the $\mathbf{1 5}$ representation of $S O(5,1)$, i.e. a field $B_{\alpha}{ }^{\beta}$ with $B_{\alpha}{ }^{\alpha}=0$, which is an $R$-symmetry scalar. Infinitesimal gauge transformations with a one-form parameter $\Lambda^{\alpha \beta}=-\Lambda^{\beta \alpha}$ act as

$$
\delta B_{\alpha}{ }^{\beta}=\partial_{\alpha \gamma} \Lambda^{\gamma \beta}-\frac{1}{4} \delta_{\alpha}{ }^{\beta} \partial_{\delta \gamma} \Lambda^{\gamma \delta},
$$

where $\partial_{\alpha \beta}=\partial_{[\alpha \beta]}$ are the space-time derivatives. For convenience, we also define a derivative with superscript indices through $\partial^{\alpha \beta} \equiv \frac{1}{2} \epsilon^{\alpha \beta \gamma \delta} \partial_{\gamma \delta}$. The gauge transformations leave the following field strength components invariant:

$$
\begin{aligned}
& H_{\alpha \beta}=\partial_{\alpha \gamma} B_{\beta}^{\gamma}+\partial_{\beta \gamma} B_{\alpha}{ }^{\gamma} \\
& H^{\alpha \beta}=\partial^{\alpha \gamma} B_{\gamma}{ }^{\beta}+\partial^{\beta \gamma} B_{\gamma}{ }^{\alpha},
\end{aligned}
$$

corresponding to the self-dual and the anti self-dual three-form representations of $S O(5,1)$ respectively. Actually, the tensor multiplet only contains the self-dual part $H_{\alpha \beta}$ of the field strength. However, it is not possible to write down an action for a self-dual three-form [7, so in order to give a Lagrangian description of it, we relax the self-duality requirement by including also the anti self-dual part $H^{\alpha \beta}$ as a "spectator field" in the theory. This means that $H^{\alpha \beta}$ in no way mixes with the other fields of the tensor multiplet. When including string interactions, the coefficients of the coupling terms in the action are to be chosen such that this field does not couple.

The derivatives of the field strength components are related by the Bianchi identity

$$
\partial^{\alpha \gamma} H_{\alpha \beta}-\partial_{\alpha \beta} H^{\alpha \gamma}=0
$$

which is easily verified by insertion.

The gauge field and the field strengths obey the reality conditions

$$
\begin{aligned}
B_{\alpha}^{* \beta} & =C_{\alpha}{ }^{\gamma}\left(-C^{*}{ }_{\delta}^{\beta}\right) B_{\gamma}{ }^{\delta} \\
H_{\alpha \beta}^{*} & =C_{\alpha}{ }^{\gamma} C_{\beta}{ }^{\delta} H_{\gamma \delta} \\
H^{* \alpha \beta} & =\left(-C^{*}{ }_{\gamma}^{\alpha}\right)\left(-C^{*}{ }_{\delta}^{\beta}\right) H^{\gamma \delta}
\end{aligned}
$$

in analogy with the conditions (17) and (9) above. 
We should also note how the fields of the tensor multiplet transform under the $R$-symmetry group $G \simeq S O(5)$. For $g \in G$, the fields transform as

$$
\begin{aligned}
\Psi_{\alpha} & \rightarrow g \Psi_{\alpha} \\
\Phi & \rightarrow g \Phi g^{T} \\
B_{\alpha}{ }^{\beta} & \rightarrow B_{\alpha}{ }^{\beta} .
\end{aligned}
$$

Since $\Omega$ is an $S O(5)$-invariant matrix, we also have the transformation

$$
\Omega \rightarrow g \Omega g^{T}=\Omega .
$$

The supersymmetric and $S O(5)$ invariant action governing the dynamics of the free $(2,0)$ tensor multiplet is

$$
S=\int d^{6} x\left\{\operatorname{Tr}\left(\partial_{\alpha \beta} \Phi \partial^{\alpha \beta} \Phi^{*}\right)+2 H_{\alpha \beta} H^{\alpha \beta}-4 i \Psi_{\alpha}^{T} \Omega \partial^{\alpha \beta} \Psi_{\beta}\right\},
$$

where we, as noted above, are forced to include the anti self-dual part of $H$. Under arbitrary variations of the fields $B_{\alpha}{ }^{\beta}, \Phi$ and $\Psi_{\alpha}$, the action yields the equations of motion

$$
\begin{aligned}
\partial^{\alpha \beta} \partial_{\alpha \beta} \Phi & =0 \\
\partial^{\alpha \gamma} H_{\alpha \beta}+\partial_{\alpha \beta} H^{\alpha \gamma} & =0 \\
\partial^{\alpha \beta} \Psi_{\beta} & =0 .
\end{aligned}
$$

In [8], these are obtained by a superspace approach at the level of equations of motion.

\subsection{The supersymmetry transformations}

The supersymmetry transformations of the fields in the tensor multiplet are

$$
\begin{aligned}
\delta \Phi & =i \eta^{\alpha} \Psi_{\alpha}^{T}+i \Psi_{\alpha} \eta^{T^{\alpha}}+\frac{i}{2}\left(\eta^{T \alpha} \Omega \Psi_{\alpha}\right) \Omega \\
\delta B_{\alpha}{ }^{\beta} & =i \eta^{T} \Omega \Psi_{\alpha}-\frac{i}{4} \delta_{\alpha}{ }^{\beta} \eta^{T \gamma} \Omega \Psi_{\gamma} \\
\delta \Psi_{\alpha} & =H_{\alpha \beta} \eta^{\beta}+2 \partial_{\alpha \beta} \Phi \Omega \eta^{\beta},
\end{aligned}
$$

where $\eta^{\alpha}$ is a constant fermionic parameter. The variations of the fields obey the same reality and $S O(5)$ properties as the fields themselves. The resulting transformation of the self-dual field strength becomes

$$
\delta H_{\alpha \beta}=i \eta^{T \gamma} \Omega\left(\partial_{\alpha \gamma} \Psi_{\beta}+\partial_{\beta \gamma} \Psi_{\alpha}\right) .
$$

It is also worthwhile to compute the transformation of the anti self-dual part of the field strength

$$
\delta H^{\alpha \beta}=i \eta^{T \alpha} \Omega \partial^{\beta \gamma} \Psi_{\gamma}+i \eta^{T \beta} \Omega \partial^{\alpha \gamma} \Psi_{\gamma}
$$


It follows from Eq. (30) that this variation vanishes when imposing the equations of motion for $\Psi$. We also note that $H^{\alpha \beta}$ does not appear in the right-hand sides of Eqs. (31) - (33). This shows that $H^{\alpha \beta}$ is not really part of the tensor multiplet.

One may verify that the supersymmetry algebra closes on-shell, i.e. the commutator of two supersymmetry transformations yields a translation according to

$$
\left[\delta_{1}, \delta_{2}\right]=2 i \eta_{1}^{T \alpha} \Omega \eta_{2}^{\beta} \partial_{\alpha \beta}
$$

when acting on any of the fields in the tensor multiplet. Two supersymmetry transformations acting on the anti self-dual part $H^{\alpha \beta}$ of the field strength is zero on-shell.

If we perform a local supersymmetry variation of the action (27) we obtain

$$
\delta S=\int d^{6} x\left(i \partial^{\alpha \beta} \eta^{\gamma}(x)\right)^{T} \Omega\left\{8 H_{\gamma \alpha} \Psi_{\beta}-16 \partial_{\gamma \alpha} \Phi \Omega \Psi_{\beta}\right\}
$$

This means that the Noether currents $\left(Q_{\gamma}\right)_{\alpha \beta}$ corresponding to the supersymmetries are given by

$$
\left(Q_{\gamma}\right)_{\alpha \beta}=8 H_{\gamma[\alpha} \Psi_{\beta]}-16 \partial_{\gamma[\alpha} \Phi \Omega \Psi_{\beta]}
$$

and are conserved, i.e. $\partial^{\alpha \beta}\left(Q_{\gamma}\right)_{\alpha \beta}=0$ when the equations of motion are imposed together with the Bianchi identity.

\section{Spontaneous symmetry breaking}

We next consider a configuration where the $R$-symmetry group is spontaneously broken from $S O(5)$ to $S O(4)$ by the expectation values of the scalar fields $\Phi$, which are the moduli of the model. This breaks four continuous symmetries and therefore gives rise to four Goldstone bosons. We will mainly follow the procedure outlined in Ch. 19 of 9 .

Since $\Phi$ has five linearly independent components, we may think of it as a vector in a five-dimensional space. An $S O(5)$ transformation rotates this vector. By giving the components of $\Phi$ non-zero vacuum expectation values we choose a direction in this space, which breaks the $S O(5)$ symmetry spontaneously to $S O(4)$ (rotations in the transverse space). This means that the $S O(5)$ symmetry will be realized non-linearly, and the coset space $S O(5) / S O(4)$ is parametrized by the Goldstone bosons.

\subsection{The action}

Mathematically, the symmetry breaking is imposed by choosing a fixed matrix $\hat{\Phi}$ in the 5 representation of $G \simeq S O(5)$ such that $-\frac{1}{4} \operatorname{Tr}\left(\hat{\Phi} \hat{\Phi}^{*}\right)=1$, and defining a subgroup $H \simeq S O(4)$ in $G$ by requiring that $h \hat{\Phi} h^{T}=\hat{\Phi}$ for all $h \in H$. This corresponds to choosing a fixed "direction" for the expectation values of the $\Phi$ field. Since $H \simeq$ $S O(4) \simeq S U(2) \times S U(2)$, all matrices in $H$ are block-diagonal with two $S U(2)$ matrices 
as diagonal elements. Explicitly, we choose our coordinates such that

$$
\hat{\Phi}=\left(\begin{array}{cc}
\sigma_{2} & \mathbf{0} \\
\mathbf{0} & -\sigma_{2}
\end{array}\right)=\left(\begin{array}{cccc}
0 & -i & 0 & 0 \\
i & 0 & 0 & 0 \\
0 & 0 & 0 & i \\
0 & 0 & -i & 0
\end{array}\right)
$$

in the basis where the $S O(5)$ invariant matrix $\Omega$ is given by Eq. (5).

Now define the right coset space $G / H$ by identifying the group element $g \in G$ with $g h$ for $h \in H$. Let $\gamma=\gamma(\xi)$ be a representative element in this four-dimensional space, which is parametrized by the Goldstone boson fields $\xi$. Any $\Phi$ in the $\mathbf{5}$ irrep of $G$ may now be written as

$$
\Phi=\phi \gamma \hat{\Phi} \gamma^{T}
$$

where the real valued field $\phi$ is given by $\phi^{2}=-\frac{1}{4} \operatorname{Tr}\left(\Phi \Phi^{*}\right)$. (Note that $\gamma \gamma^{\dagger}=\gamma^{\dagger} \gamma=\mathbf{1}_{4}$, i.e. $\gamma$ is a unitary matrix.) Hence, the vacuum expectation value $\langle\phi\rangle$ is a measure of how far we are from the origin of the moduli space and therefore sets the scale of the symmetry breaking. Analogously, we define a new spinor field $\widetilde{\Psi}$ by

$$
\Psi=\gamma \widetilde{\Psi}
$$

This field transforms linearly under $H \simeq S O(4)$, i.e. $\widetilde{\Psi} \rightarrow h \widetilde{\Psi}$ for $h \in H$, and nonlinearly under the full $G \simeq S O(5)$ group.

We may now insert the expressions (40) and (41) in the action (27). Starting with the kinetic term for the fermionic field, we get

$$
-4 i \Psi_{\alpha}^{T} \Omega \partial^{\alpha \beta} \Psi_{\beta}=-4 \widetilde{\Psi}_{\alpha}^{T} \Omega\left(i \partial^{\alpha \beta}+i \gamma^{-1} \partial^{\alpha \beta} \gamma\right) \widetilde{\Psi}_{\beta}
$$

The Lie algebra element $i \gamma^{-1} \partial^{\alpha \beta} \gamma$ may be decomposed according to

$$
i \gamma^{-1} \partial^{\alpha \beta} \gamma=A^{\alpha \beta}+\Pi^{\alpha \beta}
$$

where $A^{\alpha \beta}$ consists of the part belonging to the Lie algebra of $H$, and $\Pi^{\alpha \beta}$ belongs to its orthogonal complement in the Lie algebra of $G$. In other words, $A^{\alpha \beta}$ is the block-diagonal part and $\Pi^{\alpha \beta}$ is off-block-diagonal. Actually, $\Pi^{\alpha \beta}$ is the covariant derivative of the Goldstone fields. It is useful to note that $A^{\alpha \beta}$ commutes with the matrix $\hat{\Phi} \Omega=\Omega \hat{\Phi}$, while $\Pi^{\alpha \beta}$ anti-commutes.

Using this expansion, we may introduce a covariant derivative $D^{\alpha \beta}$ which acts on an $S O(4)$ covariant spinor according to

$$
D^{\alpha \beta} \widetilde{\Psi}_{\beta}=\left(\partial^{\alpha \beta}-i A^{\alpha \beta}\right) \widetilde{\Psi}_{\beta}
$$

this quantity transforms in the same way as $\widetilde{\Psi}$ under a $G$ transformation. 
Expanding the kinetic term for the $\Phi$ field in the same way, the action in the new set of variables becomes

$$
\begin{aligned}
S= & \int d^{6} x\left\{-4 \partial_{\alpha \beta} \phi \partial^{\alpha \beta} \phi-4 \phi^{2} \operatorname{Tr}\left(\Pi_{\alpha \beta} \Pi^{\alpha \beta}\right)+2 H_{\alpha \beta} H^{\alpha \beta}-\right. \\
& \left.-4 i \widetilde{\Psi}_{\alpha}^{T} \Omega D^{\alpha \beta} \widetilde{\Psi}_{\beta}-4 \widetilde{\Psi}_{\alpha}^{T} \Omega \Pi^{\alpha \beta} \widetilde{\Psi}_{\beta}\right\} .
\end{aligned}
$$

This is the form of the action that we intend to use when coupling the tensor multiplet to a string. The action is manifestly $S O(4)$ invariant, but $S O(5)$ invariance in ensured by the use of covariant derivatives.

By varying this action with respect to $B, \phi, \widetilde{\Psi}$ and the Goldstone boson fields $\xi$, we obtain the equations of motion

$$
\begin{aligned}
\partial_{\alpha \beta} \partial^{\alpha \beta} \phi-\phi \operatorname{Tr}\left(\Pi_{\alpha \beta} \Pi^{\alpha \beta}\right) & =0 \\
\phi D_{\alpha \beta} \Pi^{\alpha \beta}+2 \partial_{\alpha \beta} \phi \Pi^{\alpha \beta} & =0 \\
\partial^{\alpha \gamma} H_{\alpha \beta}+\partial_{\alpha \beta} H^{\alpha \gamma} & =0 \\
\left(i D^{\alpha \beta}+\Pi^{\alpha \beta}\right) \widetilde{\Psi}_{\beta} & =0 .
\end{aligned}
$$

The equations of motion can also be obtained by a change of variables in Eqs. (28) (30). Note that the first two equations above both follow from Eq. (28).

\subsection{The supersymmetry transformations}

The next step is to derive the supersymmetry transformations of $H, \phi, \Pi$ and $\widetilde{\Psi}$. For this purpose, we define $\tilde{\eta}$ through

$$
\eta=\gamma \tilde{\eta}
$$

The transformation of $H$ follows from a simple rewriting of Eq. (34) and becomes

$$
\delta H_{\alpha \beta}=\tilde{\eta}^{T \gamma} \Omega\left(\left(i D_{\alpha \gamma}+\Pi_{\alpha \gamma}\right) \widetilde{\Psi}_{\beta}+\left(i D_{\beta \gamma}+\Pi_{\beta \gamma}\right) \widetilde{\Psi}_{\alpha}\right),
$$

while the anti self-dual part transforms as

$$
\delta H^{\alpha \beta}=\tilde{\eta}^{T \alpha} \Omega\left(i D^{\beta \gamma}+\Pi^{\beta \gamma}\right) \widetilde{\Psi}_{\gamma}+\tilde{\eta}^{T \beta} \Omega\left(i D^{\alpha \gamma}+\Pi^{\alpha \gamma}\right) \widetilde{\Psi}_{\gamma}
$$

The transformation of $\phi$ follows from the equation $\phi^{2}=-\frac{1}{4} \operatorname{Tr}\left(\Phi \Phi^{*}\right)$ combined with Eq. (31). The result is

$$
\delta \phi=-\frac{i}{2} \tilde{\eta}^{T \alpha} \hat{\Phi} \widetilde{\Psi}_{\alpha}
$$

To proceed, we need to consider the supersymmetry transformation of $i \gamma^{-1} \partial^{\alpha \beta} \gamma$. This obeys the identity

$$
\delta\left(i \gamma^{-1} \partial^{\alpha \beta} \gamma\right)=i\left[\left(i \gamma^{-1} \delta \gamma\right),\left(i \gamma^{-1} \partial^{\alpha \beta} \gamma\right)\right]+\partial^{\alpha \beta}\left(i \gamma^{-1} \delta \gamma\right)
$$


In analogy with the decomposition (43) we write

$$
i \gamma^{-1} \delta \gamma=S+T
$$

where $S$ denotes the block-diagonal part and $T$ the off-block-diagonal part. By transforming both sides of Eq. (40) and comparing with Eq. (31), we find that

$$
T=-\frac{1}{4 \phi}\left(\left(\tilde{\eta}^{\gamma} \widetilde{\Psi}_{\gamma}^{T}+\widetilde{\Psi}_{\gamma} \tilde{\eta}^{T \gamma}\right) \hat{\Phi}-\Omega \hat{\Phi}\left(\tilde{\eta}^{\gamma} \widetilde{\Psi}_{\gamma}^{T}+\widetilde{\Psi}_{\gamma} \tilde{\eta}^{T \gamma}\right) \Omega\right)
$$

An explicit expression for $S$ is never needed in the calculations.

Hence, using Eq. (43), we may decompose Eq. (54) as

$$
\begin{aligned}
\delta A^{\alpha \beta} & =i\left[S, A^{\alpha \beta}\right]+i\left[T, \Pi^{\alpha \beta}\right]+\partial^{\alpha \beta} S \\
\delta \Pi^{\alpha \beta} & =i\left[T, A^{\alpha \beta}\right]+i\left[S, \Pi^{\alpha \beta}\right]+\partial^{\alpha \beta} T .
\end{aligned}
$$

It is convenient to introduce a covariant supersymmetry variation $\Delta$ acting as

$$
\begin{aligned}
\Delta \widetilde{\Psi}_{\alpha} & =\delta \widetilde{\Psi}_{\alpha}-i S \widetilde{\Psi}_{\alpha} \\
\Delta \Pi^{\alpha \beta} & =\delta \Pi^{\alpha \beta}-i\left[S, \Pi^{\alpha \beta}\right] .
\end{aligned}
$$

This yields that

$$
\Delta \Pi^{\alpha \beta}=\partial^{\alpha \beta} T-i\left[A^{\alpha \beta}, T\right] \equiv D^{\alpha \beta} T,
$$

where we have introduced the covariant derivative acting on $T$. We also find that

$$
\Delta \widetilde{\Psi}_{\alpha}=i T \widetilde{\Psi}_{\alpha}+H_{\alpha \beta} \tilde{\eta}^{\beta}+2 \partial_{\alpha \beta} \phi \hat{\Phi} \Omega \tilde{\eta}^{\beta}+4 i \phi \hat{\Phi} \Omega \Pi_{\alpha \beta} \tilde{\eta}^{\beta}
$$

and

$$
\Delta\left(D^{\alpha \beta} \widetilde{\Psi}_{\gamma}\right)=D^{\alpha \beta}\left(\Delta \widetilde{\Psi}_{\gamma}\right)+\left[T, \Pi^{\alpha \beta}\right] \widetilde{\Psi}_{\gamma}
$$

Having found the supersymmetry transformations of the fields, we now consider the action (45). It is straightforward to verify that

$$
\begin{aligned}
& \delta \operatorname{Tr}\left(\Pi_{\alpha \beta} \Pi^{\alpha \beta}\right)=2 \operatorname{Tr}\left(\Delta \Pi_{\alpha \beta} \Pi^{\alpha \beta}\right) \\
& \delta\left(\widetilde{\Psi}_{\alpha}^{T} \Omega D^{\alpha \beta} \widetilde{\Psi}_{\beta}\right)=\left(\Delta \widetilde{\Psi}_{\alpha}\right)^{T} \Omega D^{\alpha \beta} \widetilde{\Psi}_{\beta}+\widetilde{\Psi}_{\alpha}^{T} \Omega \Delta\left(D^{\alpha \beta} \widetilde{\Psi}_{\beta}\right) \\
& \delta\left(\widetilde{\Psi}_{\alpha}^{T} \Omega \Pi^{\alpha \beta} \widetilde{\Psi}_{\beta}\right)=2 \widetilde{\Psi}_{\alpha}^{T} \Omega \Pi^{\alpha \beta} \Delta \widetilde{\Psi}_{\beta}+\widetilde{\Psi}_{\alpha}^{T} \Omega\left(\Delta \Pi^{\alpha \beta}\right) \widetilde{\Psi}_{\beta},
\end{aligned}
$$

by using that $\operatorname{Tr}([S, \Pi] \Pi)=0$ and that $S^{T} \Omega+\Omega S=0$. It follows that the action (45) is, as expected, invariant under the supersymmetry transformations. In this calculation, it is necessary to use that

$$
\begin{aligned}
D_{\alpha \beta} \Pi^{\alpha \gamma} & =\frac{1}{4} \delta_{\beta}^{\gamma} D_{\alpha \delta} \Pi^{\alpha \delta} \\
\Pi_{\alpha \beta} \Pi^{\alpha \beta} & =\frac{1}{4} \operatorname{Tr}\left(\Pi_{\alpha \beta} \Pi^{\alpha \beta}\right) \mathbf{1}_{4} .
\end{aligned}
$$


We also apply the fact that the supersymmetry transformation is global, i.e. $\partial \eta=0$, which gives that

$$
D_{\alpha \beta} \tilde{\eta}^{\beta}=i \Pi_{\alpha \beta} \tilde{\eta}^{\beta} .
$$

For future reference, we note that a local supersymmetry variation of the action yields the Noether currents

$$
\left(\tilde{Q}_{\gamma}\right)_{\alpha \beta}=8 H_{\gamma[\alpha} \widetilde{\Psi}_{\beta]}-32 i \phi \hat{\Phi} \Omega \Pi_{\gamma[\alpha} \widetilde{\Psi}_{\beta]}-16 \partial_{\gamma[\alpha} \phi \hat{\Phi} \Omega \widetilde{\Psi}_{\beta]},
$$

obeying the conservation equation

$$
\left(i D^{\alpha \beta}+\Pi^{\alpha \beta}\right)\left(\tilde{Q}_{\gamma}\right)_{\alpha \beta}=0 .
$$

\section{The free string}

\subsection{The fields}

A configuration containing a straight static string breaks translational invariance in the directions transverse to the string and also half of the supersymmetries. The corresponding Goldstone modes consist of four bosons transforming as $(\mathbf{2}, \mathbf{2} ; \mathbf{1}, \mathbf{1})$, four left moving fermions transforming as $(\mathbf{2}, \mathbf{1} ; \mathbf{2}, \mathbf{1})$, and four right moving fermions transforming as $(\mathbf{1}, \mathbf{2} ; \mathbf{1}, \mathbf{2})$ under $S O(4) \times S O(4) \simeq S U(2) \times S U(2) \times S U(2) \times S U(2)$. Here, the first and second $S O(4)$ factors are the unbroken parts of the Lorentz and $R$-symmetry groups respectively. Quantization of the zero-modes of these fields gives rise to the vector string multiplet described in section two. Quantization of their non zero-modes gives rise to a Fock space of waves propagating on the world-sheet of the string.

To construct such a string theory, it is convenient to supplement these worldsheet Goldstone fields with extra components so that they fill out representations of the unbroken Lorentz group $S O(5,1)$. We will thus take the fields on the string worldsheet to be a space-time vector $X^{\alpha \beta}=X^{[\alpha \beta]}$ which is an $R$-symmetry singlet, and a space-time spinor $\tilde{\theta}^{\alpha}$ in the $(\mathbf{2}, \mathbf{1}) \oplus(\mathbf{1}, \mathbf{2})$ representation of the unbroken subgroup $S O(4) \simeq S U(2) \times S U(2)$ of the $R$-symmetry group. The action governing these fields should be invariant under local reparametrizations of the world-sheet and a local fermionic ' $\kappa$-symmetry' by which the added components can be eliminated. It is also convenient to introduce an auxiliary world-sheet metric $g_{i j}$, where $i, j=1,2$ are world-sheet vector indices. This field obeys an algebraic equation of motion [10, 11], and thus does not contain any independent degrees of freedom.

The action should be invariant under all transformations of the $(2,0)$ superalgebra. However, only the unbroken $S O(4)$ subgroup of the $R$-symmetry group will be linearly realized. The full $S O(5) R$-symmetry group can then be non-linearly realized by replacing all derivatives with covariant derivatives involving the space-time Goldstone fields discussed in section four. We will not discuss such couplings between spacetime and world-sheet fields in this paper, though, but defer them to a forthcoming publication. This also applies to the string tension, which we will simply take to be 
given by the vacuum expectation value $\langle\phi\rangle$ of $\phi$, although it should be given by the local value of $\phi$ in the complete theory.

\subsection{The action and its symmetries}

The construction of the theory is largely analogous to the Green-Schwarz superstring [12]. Global supersymmetry transformations act as

$$
\begin{aligned}
\delta \tilde{\theta}^{\alpha} & =\tilde{\eta}^{\alpha} \\
\delta X^{\alpha \beta} & =i \tilde{\eta}^{T[\alpha} \Omega \tilde{\theta}^{\beta]} \\
\delta g_{i j} & =0
\end{aligned}
$$

where we take the parameters $\tilde{\eta}^{\alpha}$ to be constant. In the complete theory, they should fulfill a requirement like Eq. (69). We define a 'conjugate momentum' as

$$
P_{i}^{\alpha \beta}=\partial_{i} X^{\alpha \beta}-i \tilde{\theta}^{T[\alpha} \Omega \partial_{i} \tilde{\theta}^{\beta]}
$$

and find that

$$
\delta P_{i}^{\alpha \beta}=0 .
$$

Denoting the world-sheet coordinates by $\sigma^{i}$, the term

$$
S_{1}=-\frac{1}{8}\langle\phi\rangle \int d^{2} \sigma \sqrt{g} g^{i j} \epsilon_{\alpha \beta \gamma \delta} P_{i}^{\alpha \beta} P_{j}^{\gamma \delta},
$$

where $g^{i j}$ is the inverse of $g_{i j}$ and $g=-\operatorname{det} g_{i j}$, is obviously invariant under supersymmetry. We also consider the term

$$
S_{2}=-\frac{1}{4}\langle\phi\rangle \int d^{2} \sigma \epsilon^{i j} \epsilon_{\alpha \beta \gamma \delta}\left(i P_{i}^{\alpha \beta} \tilde{\theta}^{T \gamma} \hat{\Phi} \partial_{j} \tilde{\theta}^{\delta}-\frac{1}{2} \tilde{\theta}^{T \alpha} \Omega \partial_{i} \tilde{\theta}^{\beta} \tilde{\theta}^{T \gamma} \hat{\Phi} \partial_{j} \tilde{\theta}^{\delta}\right) .
$$

Using the constancy of the parameters $\tilde{\eta}^{\alpha}$ and discarding total derivatives, it is straightforward to show that

$$
\delta S_{2}=\frac{1}{4}\langle\phi\rangle \int d^{2} \sigma \epsilon^{i j} \epsilon_{\alpha \beta \gamma \delta} \lambda_{[1}^{T \alpha} \Omega \lambda_{2}^{\beta} \lambda_{3}^{T \gamma} \hat{\Phi} \lambda_{4]}^{\delta},
$$

where $\lambda_{1}^{\alpha}=\tilde{\eta}^{\alpha}, \lambda_{2}^{\beta}=\tilde{\theta}^{\beta}, \lambda_{3}^{\gamma}=i \partial_{i} \tilde{\theta}^{\gamma}, \lambda_{4}^{\delta}=i \partial_{j} \tilde{\theta}^{\delta}$. But by direct computation, one can verify the identity

$$
v_{[1}^{T} \Omega v_{2} v_{3}^{T} \hat{\Phi} v_{4]}=0
$$

which is valid for any Grassmann even quantities $v_{1}, \ldots, v_{4}$ in the $(\mathbf{2}, \mathbf{1}) \oplus(\mathbf{1}, \mathbf{2})$ representation of $S O(4)$. It follows that the integrand of Eq. (79), which involves Grassmann odd quantities $\lambda_{1}^{\alpha}, \ldots, \lambda_{4}^{\delta}$, vanishes because of the antisymmetry of $\epsilon_{\alpha \beta \gamma \delta}$. So the term $S_{2}$ is invariant under supersymmetry.

The local fermionic symmetry has parameters $\tilde{\kappa}_{\alpha}^{i}$ that are functions of the worldsheet coordinates $\sigma^{i}$. They are subject to the constraint

$$
\tilde{\kappa}_{\alpha}^{i}=\mathcal{P}^{i j} g_{j k} \tilde{\kappa}_{\alpha}^{k}
$$


where the projection operator $\mathcal{P}^{i j}$ is given by

$$
\mathcal{P}^{i j}=\frac{1}{2}\left(g^{i j} \mathbf{1}_{4}-\epsilon^{i j} \Omega \hat{\Phi} / \sqrt{g}\right) .
$$

The transformations act on the fields as

$$
\begin{aligned}
\delta_{\kappa} \tilde{\theta}^{\alpha} & =P_{i}^{\alpha \beta} \tilde{\kappa}_{\beta}^{i} \\
\delta_{\kappa} X^{\alpha \beta} & =-i \delta_{\kappa} \tilde{\theta}^{T[\alpha} \Omega \tilde{\theta}^{\beta]} \\
\delta_{\kappa}\left(\sqrt{g} g^{i j}\right) & =2 i \sqrt{g}\left(\mathcal{P}^{i k} \tilde{\kappa}_{\alpha}^{j}\right)^{T} \Omega \partial_{k} \tilde{\theta}^{\alpha} .
\end{aligned}
$$

The last equation is consistent with the symmetry and unimodularity of $\sqrt{g} g^{i j}$. It follows from the first two equations that

$$
\delta_{\kappa} P_{i}^{\alpha \beta}=-2 i \delta_{\kappa} \tilde{\theta}^{T[\alpha} \Omega \partial_{i} \tilde{\theta}^{\beta]} .
$$

The terms $S_{1}$ and $S_{2}$ are not separately invariant under these transformations. However, by using Eq. (80), one can verify that the variation of the linear combination

$$
S=S_{1}+S_{2}
$$

vanishes. This is our free string action. It should however be coupled to a space-time tensor multiplet so that the string tension is given by the local value of $\phi$ rather than by $\langle\phi\rangle$, and so that ordinary derivatives are replaced by covariant derivatives involving the pullback of the space-time Goldstone fields to the world-sheet. The string should also couple electrically and magnetically to the two-form gauge field of the tensor multiplet. Constructing such a theory while maintaining supersymmetry and $\kappa$-symmetry is a challenging problem, on which we hope to make further progress.

Acknowledgments: M.H. is a Research Fellow at the Royal Swedish Academy of Sciences. He would like to thank the Institute for Advanced Study in Princeton for its hospitality, and Edward Witten for an illuminating discussion on spontaneous symmetry breaking. We would also like to thank Martin Cederwall for many helpful discussions on supersymmetry. 


\section{References}

[1] P. Arvidsson, E. Flink and M. Henningson, Thomson scattering of chiral tensors and scalars against a self-dual string, JHEP 12 (2002) 010 hep-th/0210223.

[2] E. Witten, Some comments on string dynamics, hep-th/9507121.

[3] W. Nahm, Supersymmetries and their representations, Nucl. Phys. B135 (1978) 149.

[4] T. Kugo and P. K. Townsend, Supersymmetry and the division algebras, Nucl. Phys. B221 (1983) 357.

[5] P. S. Howe, N. D. Lambert and P. C. West, The self-dual string soliton, Nucl. Phys. B515 (1998) 203-216 hep-th/9709014.

[6] A. Gustavsson and M. Henningson, A short representation of the six-dimensional (2,0) algebra, JHEP 06 (2001) 054 hep-th/0104172.

[7] E. Witten, Five-brane effective action in M-theory, J. Geom. Phys. 22 (1997) 103-133 hep-th/9610234.

[8] P. S. Howe, G. Sierra and P. K. Townsend, Supersymmetry in six-dimensions, Nucl. Phys. B221 (1983) 331.

[9] S. Weinberg, The quantum theory of fields, vol. II. Cambridge University Press, 1996.

[10] L. Brink, P. Di Vecchia and P. S. Howe, A locally supersymmetric and reparametrization invariant action for the spinning string, Phys. Lett. B65 (1976) 471-474.

[11] S. Deser and B. Zumino, A complete action for the spinning string, Phys. Lett. B65 (1976) 369-373.

[12] M. B. Green and J. H. Schwarz, Covariant description of superstrings, Phys. Lett. B136 (1984) 367-370. 